\title{
Nematic order in square lattice frustrated ferromagnets
}

\author{
Nic Shannon, ${ }^{1,2}$ Tsutomu Momoi, ${ }^{3}$ and Philippe Sindzingre ${ }^{4}$ \\ ${ }^{1}$ Max-Planck-Institut für Chemische Physik fester Stoffe, Nöthnitzer Str. 40, 01187 Dresden, Germany \\ ${ }^{2}$ H. H. Wills Physics Laboratory, University of Bristol, Tyndall Ave, BS8-1TL, UK \\ ${ }^{3}$ Condensed Matter Theory Laboratory, RIKEN, Wako, Saitama 351-0198, Japan \\ ${ }^{4}$ Laboratoire de Physique Théorique de la Matière Condensée, UMR 7600 of CNRS, \\ Université P. et M. Curie, case 121, 4 Place Jussieu, 75252 Paris Cedex, France
}

(Dated: April 2, 2018)

\begin{abstract}
We present a new scenario for the breakdown of ferromagnetic order in a two-dimensional quantum magnet with competing ferromagnetic and antiferromagnetic interactions. In this, dynamical effects lead to the formation of two-magnon bound states, which undergo Bose-Einstein condensation, giving rise to bond-centered nematic order. This scenario is explored in some detail for an extended Heisenberg model on a square lattice. In particular, we present numerical evidence confirming the existence of a state with d-wave nematic correlations but no long range magnetic order, lying between the saturated ferromagnetic and collinear antiferromagnetic phases of the ferromagnetic $J_{1}-J_{2}$ model. We argue by continuity of spectra that this phase is also present in a model with 4-spin cyclic exchange.

PACS numbers: 75.40.Cx 75.10.-b, 75.10.Jm,
\end{abstract}

The search for a true quantum "spin liquid" - a quantum magnet which remains disordered at the very lowest temperatures - in dimension greater than one has been central to research on quantum magnets for more than three decades. Following Anderson [1], most models of quantum spin liquids proposed to date have been based on frustrated antiferromagnetic (AF) interactions. The resulting spin liquid states involve strong singlet bonds between spins, which give rise to a gap in the spin excitation spectrum (for a review, see e.g. 2]).

None the less, the best characterized experimental realization of a quantum spin liquid is believed to occur in two-dimensional films of solid ${ }^{3} \mathrm{He}$, where the interactions between spins are predominantly ferromagnetic $(\mathrm{FM})$, and the resulting state is gapless [3, 4]. This raises the interesting question of whether the breakdown of long ranged FM order offers a new route to a spin liquid ground state?

The purpose of this paper is to demonstrate that a gapless spin liquid can indeed occur in a simple twodimensional model of quantum spins on a square lattice with predominantly FM interactions. To this end we present a set of analytic and numerical results which show how a new type of "bond-nematic" state emerges from the ruins of FM order in an extended Heisenberg model on a square lattice. Our work is inspired by a number of new FM square lattice compounds [5, 6 and has close parallels in earlier work on spin chains [7]. The special case of the multiple spin exchange model on a triangular lattice - which is believed to describe the magnetism of two-dimensional solid ${ }^{3} \mathrm{He}$ - will be dealt with elsewhere [8].

While these results are rather general, for concreteness in this Letter we consider a square lattice $S=1 / 2$ frus-

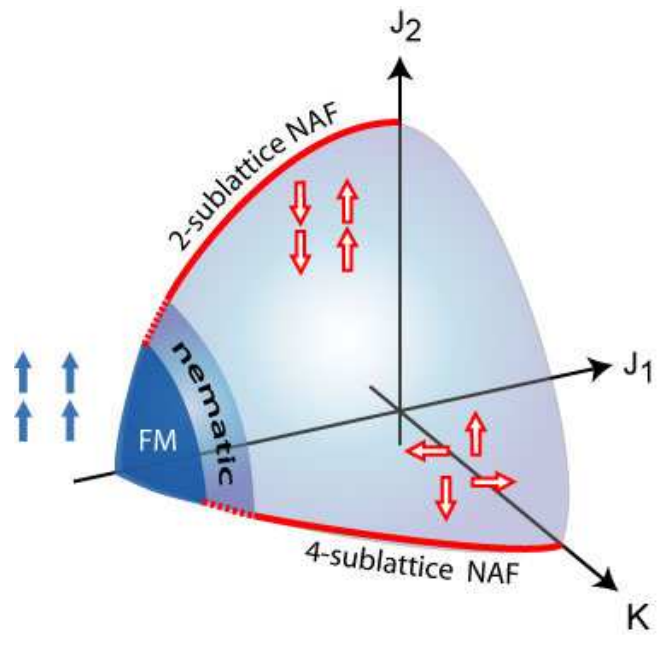

FIG. 1: Phase diagram for the spin- $1 / 2 J_{1}-J_{2}-K$ Heisenberg model on a square lattice, in the octant where $J_{1}<0$ is FM and both $J_{2} \geq 0$ and $K \geq 0$ are AF. Solid lines in the $J_{1}-J_{2}$ and $J_{1}-K$ planes denote known Néel phases. A single nematic phase interpolates between the FM occurring for large FM $J_{1}$ and these two, different, AF ordered states.

trated ferromagnet defined by the Hamiltonian

$$
\begin{aligned}
\mathcal{H}= & 2 J_{1} \sum_{\langle i j\rangle_{1}} \mathbf{S}_{i} \cdot \mathbf{S}_{j}+2 J_{2} \sum_{\langle i j\rangle_{2}} \mathbf{S}_{i} \cdot \mathbf{S}_{j} \\
& +K \sum_{\langle 1234\rangle}\left(P_{1234}+P_{1234}^{-1}\right)-h \sum_{i} S_{i}^{z},
\end{aligned}
$$

where $\langle i j\rangle_{1}$ counts nearest neighbor bonds, $\langle i j\rangle_{2}$ counts next-nearest neighbor bonds, and $P_{1234}$ performs a cyclic exchange of spins on a single square plaquette. We restrict ourselves to the case where $J_{1}<0$ is FM and the other two interactions $J_{2} \geq 0$ and $K \geq 0$ are AF.

The essence of our results, summarized in Fig. 1 and 

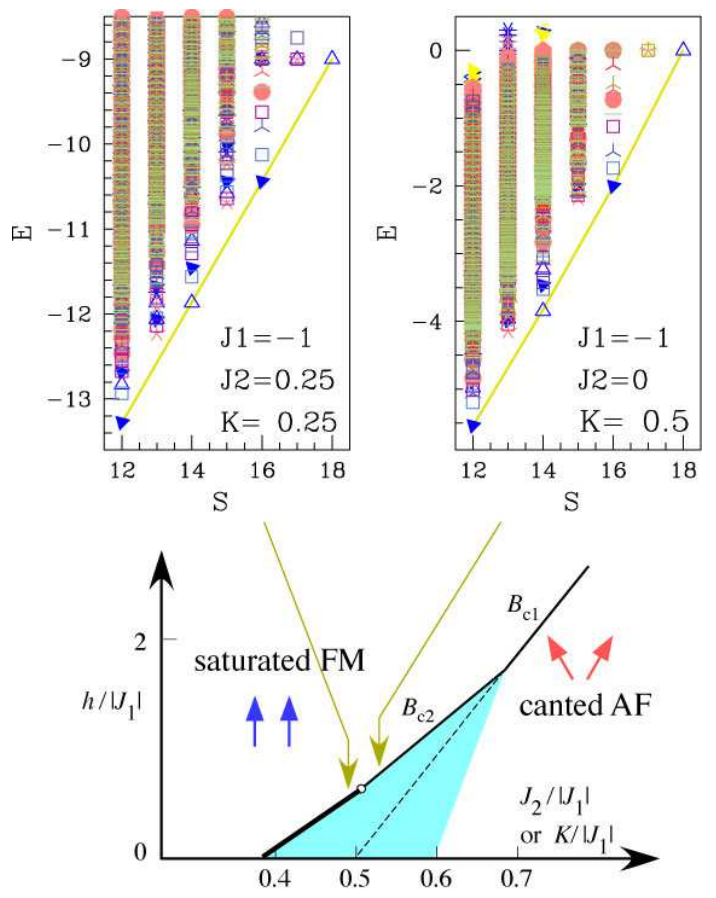

FIG. 2: Schematic phase diagram of the FM $J_{1}-J_{2}-K$ extended Heisenberg model in applied field, showing how the two-magnon instability crosses over from $1^{\text {st }}$ to $2^{\text {nd }}$ order in applied field and is eventually succeeded by a conventional one-magnon instability. The inset shows representative spectra for $1^{\text {st }}$ and $2^{\text {nd }}$ order transitions into $\mathrm{d}$-wave nematic state (high spin states only).

Fig. 2] is as follows - the competition between FM and AF interactions leads to a large "accidental" degeneracy in the one-magnon spectrum of the FM phase, which then becomes highly susceptible to the formation of twomagnon bound states. These condense, and give rise to a new singlet phase lying between the classical FM and known Néel phases of the model. This phase has a well defined Goldstone mode, but no long-range magnetic order in the conventional sense. We introduce an appropriate order parameter for this phase, and identify it as a $\mathrm{d}$-wave n-type "bond-nematic" state, distinct from conventional "n-type" and "p-type" nematic states known to occur in other closely related models [9, 10]. From numerical exact diagonalization calculations we are able both to confirm that this phase exists, and to establish that it interpolates between the two well-studied limits of Eq. (11), the square lattice $J_{1}-J_{2}$ model for $K \rightarrow 0$ and the square lattice $J_{1}-K$ multiple spin exchange model for $J_{2} \rightarrow 0$.

We now turn to the analysis. For large FM $J_{1}$ the ground state of Eq. (1) is a uniform saturated FM. In the FM $J_{1-} J_{2}$ model, for $K=0$ this gives way to $2-$ sublattice collinear Néel AF at large $J_{2}$ 11. In the FM $J_{1}-K$ model, for $J_{2}=0$, the corresponding Néel state is an orthogonal 4-sublattice structure [12]. Our analysis begins with a very simple observation - at a classical level, the transition between FM and Néel states is $2^{\text {nd }}$ order and takes place where $\left|J_{1}\right|=2\left(K+J_{2}\right)$. However, exactly at this transition, the one - magnon spectrum of the FM phase posses entire lines of zero modes [13]. This "accidental" degeneracy has profound consequences for magnetism.

Firstly, it leads to large zero point fluctuations which shift the first instability of the FM to somewhat weaker AF coupling. We have performed numerical exact diagonalization (ED) of Eq. (1) for clusters of 16, 20, 32 and 36 spins [14]. The evolution of the ground state energy indicates that a $1^{\text {st }}$ order transition from saturated FM to singlet ground states occurs for $\left|J_{1}\right| \approx 2.5\left(K+J_{2}\right)$.

Secondly, these fluctuations also destabilize the neighboring Néel phase. Semi-classical estimates for the the square lattice $J_{1}-J_{2}$ model suggest that the sublattice magnetization of its collinear Néel phase vanishes for $\left|J_{1}\right| \approx 1.97 J_{2}[13]$. These calculations almost certainly underestimate quantum effects.

These arguments provide good a priori reason to believe that the extended Heisenberg model Eq. (1) supports a new spin liquid phase for a finite range of parameters between its FM and Néel phases. However they give little insight into what this phase might be.

In order to better answer this question, we introduce magnetic field $h$ and examine the nature of the first instability of the fully saturated paramagnet as the magnetic field is reduced. The situation is summarized in Fig. 2 For parameters well within the classical Néel phases of Eq. (1), the first instability of the saturated state occurs at $h=B_{c 1}$ and is controlled by the lowest one-magnon excitation in applied field. This instability is against a conventional two-sublattice canted AF state.

In the proposed spin liquid region, however, the onemagnon spectrum is highly degenerate. In the extreme case, $J_{2}=0, K=\left|J_{1}\right| / 2$, the one-magnon dispersion vanishes altogether, so there is no net energy gain in flipping one spin at any $\mathbf{q}$ and the single flipped spins are always localized. On the other hand, pairs of flipped spins on neighboring sites can propagate coherently under the action of the cyclic exchange operator $P_{1234}$, and so gain kinetic energy. It is therefore reasonable to ask whether the transition out of the FM phase controlled by such two-magnon bound states?

Both the one-body problem of a single flipped spin$1 / 2$ in a saturated FM background $|\mathrm{FM}\rangle$ and the twobody problem of two interacting flipped spins can be solved exactly. We have calculated the energy of both states in the proposed spin liquid region and find that, at a critical value of field $h=B_{c 2}$, two-magnon bound states of the form $|\phi\rangle=\sum_{i j} \phi_{i j} S_{i}^{-} S_{j}^{-}|\mathrm{FM}\rangle$ with d-wave symmetry are gapless and give the first instability, while one-magnon states have higher positive energy - see Fig. 2 The binding energy of the two-magnon state is of purely kinetic origin. In zero field, the two-magnon 
bound states first become stable in the $J_{1}-J_{2}$ model for $J_{2} /\left|J_{1}\right|=0.408$ (c.f. [15]), and in the $J_{1}-K$ model for $K /\left|J_{1}\right|=0.364$. These values should be compared with the numerically determined boundary of the FM phase at $\left(J_{2}+K\right) /\left|J_{1}\right| \approx 0.4$. In the special case $J_{2}=0$, $K=\left|J_{1}\right| / 2$, the two-magnon bound state has the compact form $\phi_{i j}=\frac{1}{\sqrt{2}}\left[\delta_{i, j-e_{1}}-\delta_{i, j-e_{2}}\right]$, where $\mathbf{e}_{1}=(1,0)$ and $\mathbf{e}_{2}=(0,1)$ connect neighboring sites on a square lattice.

What then happens below $B_{c 2}$ ? In the case of the FM $J_{1}-K$ model for $J_{2}=0$, the answer is quite simple. Twomagnon pairs undergo Bose-Einstein condensation. For this set of parameters there is a weak, repulsive interaction between two-magnon pairs, so the transition is $2^{\text {nd }}$ order. This is evident from ED spectra - the locus of the lowest lying state in the high-spin sector has a convex curvature as a function of $S$, so the magnetization of the system must evolve continuously at $B_{c 2}$ - see the inset to Fig. 2. Even spin sector states only appear in the lowest states in magnetic field. This period two is the evidence of condensation of two-magnon pairs.

In the FM $J_{1}-J_{2}$ model for $K=0$ the situation is a little more complicated. The net interaction between two-magnon pairs is attractive, and the transition is $1^{\text {st }}$ order. Again this is evident in ED from the concave curvature of the high spin states, which signals a jump in magnetization at saturation field. A similar 1st order transition appears in the $J_{1}-K$ model for $J_{2}=0$ well close to the FM phase boundary. However in all of these cases the structure of the low lying states is essentially the same - even spin sector states always have lower energy than odd spin sector states, and show an alternation between the s-wave $A_{1}$ and d-wave $B_{1}$ irreps of the square lattice space group $C_{4 \nu}$. We return these points below.

Where bound pairs of magnons condense they give rise to a new form of bond-nematic order. This is to say that, while the orientation of individual spins remains undetermined, the traceless rank two tensor

$$
\mathcal{O}^{\alpha \beta}\left(\boldsymbol{r}_{i}, \boldsymbol{r}_{j}\right)=\frac{1}{2}\left(S_{i}^{\alpha} S_{j}^{\beta}+S_{i}^{\beta} S_{j}^{\alpha}\right)-\frac{1}{3} \delta^{\alpha \beta}\left\langle\boldsymbol{S}_{i} \cdot \boldsymbol{S}_{j}\right\rangle
$$

does exhibit long range order 16. The matrix elements of $\mathcal{O}^{\alpha \beta}\left(\boldsymbol{r}_{i}, \boldsymbol{r}_{j}\right)$ are connected to the magnon pairing operator through the relation $S_{i}^{-} S_{j}^{-}=\mathcal{O}^{x x}-\mathcal{O}^{y y}-2 i \mathcal{O}^{x y}$. Physically, one can think of this tensor operator as revealing the order "hidden" in the spin- $-1 / 2$ quantum spinliquid by projecting into a symmetrized spin-1 Hilbert space of bond variables with long range correlations.

The order parameter Eq. (2) is distinct from the sitecentred n-type nematic order seen in certain models with $S=1$ 9], and from the p-type chiral order found in the multiple spin exchange model with AF interactions [10]. However a very similar order parameter was previously introduced in the context of one-dimensional frustrated spin chains [7].

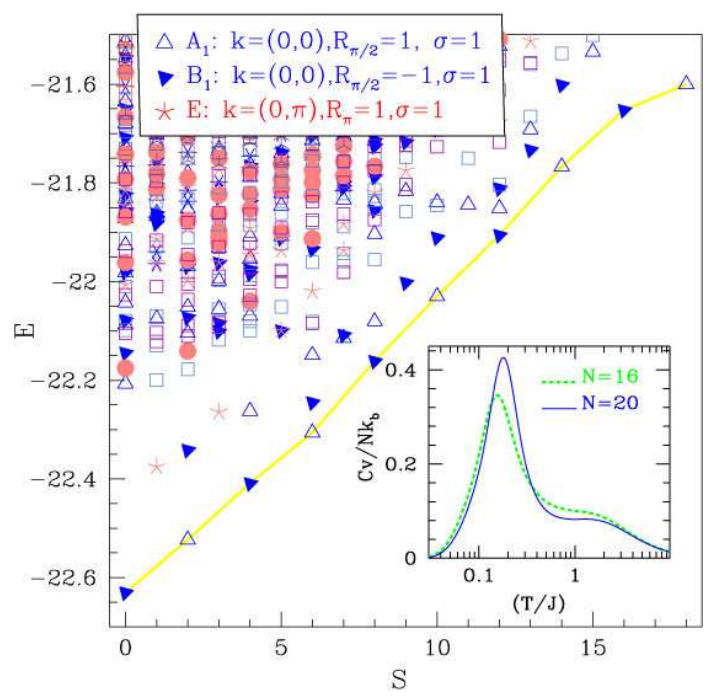

FIG. 3: Spectrum of extended Heisenberg model for $N=36$ spin cluster with $J_{1}=-1, J_{2}=0.4, K=0$, showing the marked alternation in energies of the lowest lying states in even and odd spin sectors. The inset shows the double peak structure of the heat capacity $c_{V}(T)$ for clusters of $N=16$ and $N=20$ spins and the same set of parameters.

Having established the unconventional nature of the state near saturation, we now turn to the nature of the ground state in the absence of applied field. Proof that this is a spin liquid follows from the finite-size scaling properties of ED spectra. In Fig. [3 we present the spectrum of a cluster of $N=36$ spins for $J_{1}=-1, J_{2}=0.4$ and $K=0$, very close to the boundary with the saturated FM phase. The signature of long range Néel order in such a spectrum would be the existence of a set of "quasi-degenerate joint states" (QDJS) which form the $N=\infty$ ground-sate, presents in every spin sector (up to $S \sim \sqrt{N})$, with energies scaling as $E_{\mathrm{QDJS}} \sim \frac{S(S+1)}{N}$ and are well separated (at finite $N$ ) from the lowest excitations of the continuum (magnons with energies scaling as $\left.\sim \frac{1}{\sqrt{N}}\right)$. The QDJS specific to $(\pi, 0)$ collinear AF order comprise one $A_{1}$ and one $B_{1}$ state for even $S$, and a (twofold degenerate) $E$ level for $S$ odd [17]. The gaps to the low lying states in each spin sector should evolve as $\Delta \sim \frac{1}{N}\left[\alpha-\frac{\beta}{\sqrt{N}}\right]$, with coefficients $\alpha$ and $\beta$ determined by the spin stiffness and spin wave velocity of the Néel state, and thus diplay a negative curvature when plotted against $1 / N$ [18].

It is immediately clear from the spectrum in Fig. B] that the energies of the odd spin states, which include the $E$ symmetry states, are well separated from those of the even spin states. Thus the spectrum is not compatible with the expected Néel order. The unconventional nature of the ground state is also reflected in the finite size scaling of gaps, shown in Fig. [4 The gaps in the even spin sectors scale to zero in the expected manner. 


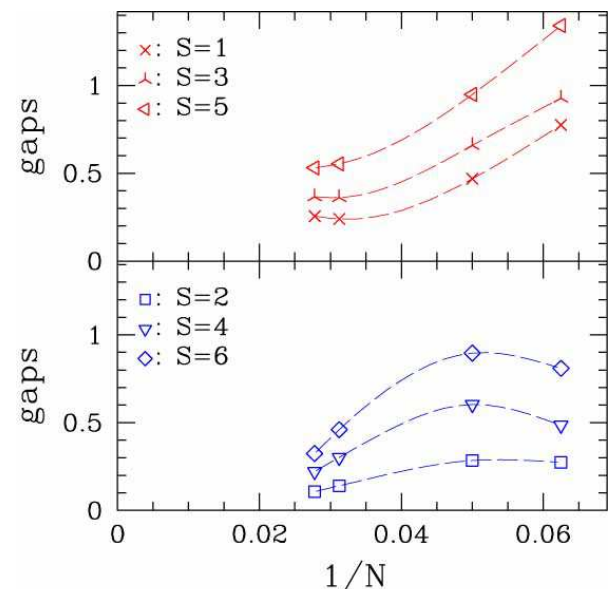

FIG. 4: Finite size scaling of the energy gaps of extended Heisenberg model with $J_{1}=-1, J_{2}=0.4, K=0$, for $A_{1}$ and $B_{1}$ symmetry states (lower) and $E$ ones (upper) in the even and odd spin sectors, respectively.

But the gaps of $E$ symmetry states in odd spin sectors do not - in fact they appear to scale to a finite value $\Delta_{\text {odd }} \sim 0.4$.

This is conclusive proof that the FM $J_{1}-J_{2}$ model does not exhibit Néel order in the immediate neighborhood of its FM phase. But we can draw a still stronger conclusion. The spin even-odd oscillation found in high spin states persists down to low spin states, and the $A_{1}$ and $B_{1}$ states found in even spin sectors are precisely the same states which emerge from the two magnon instability of the saturated FM in applied magnetic field. The existence of this set of QDJS is the signature of nematic order with $\mathrm{d}$-wave symmetry. Thus the nematic order found near to saturation persists down to zero magnetic field.

Such nematic order has a degenerate ground-state composed of even spin states. It has gapless Goldstone modes analogous to the lowest magnons states of the Néel state, present in the $S=1$ sector, scaling as $\frac{1}{\sqrt{N}}$. Results for a prototypical nematic correlation function $\sum_{\alpha, \beta}\left\langle\mathcal{O}^{\alpha \beta}\left(\mathbf{0}, \mathbf{e}_{1}\right) \mathcal{O}^{\alpha \beta}\left(\mathbf{r}, \mathbf{r}+\mathbf{e}_{a}\right)\right\rangle(a=1,2)$ in the nematic ground state are presented in Fig. 5 Nematic correlations exhibit a "stripey" character, and exist in the whole system. Because of the underlying d-wave symmetry, correlations on parallel bond have positive sign, while correlations on perpendicular bonds are negative.

The finite temperature properties of the FM $J_{1}-J_{2}$ model provides further insight into the bond-nematic state. In an inset to Fig. 3 we show the heat capacity calculated for the same exchange parameters. It exhibits two clear energy scales - a broad structure extending to $T \sim\left|J_{1}\right|$ which reflects the formation of $S=1 \mathrm{ob}-$ jects from the paramagnetic "soup", and sharp peak at $T \sim 0.1\left|J_{1}\right|$, where these $S=1$ objects start to order nematically.

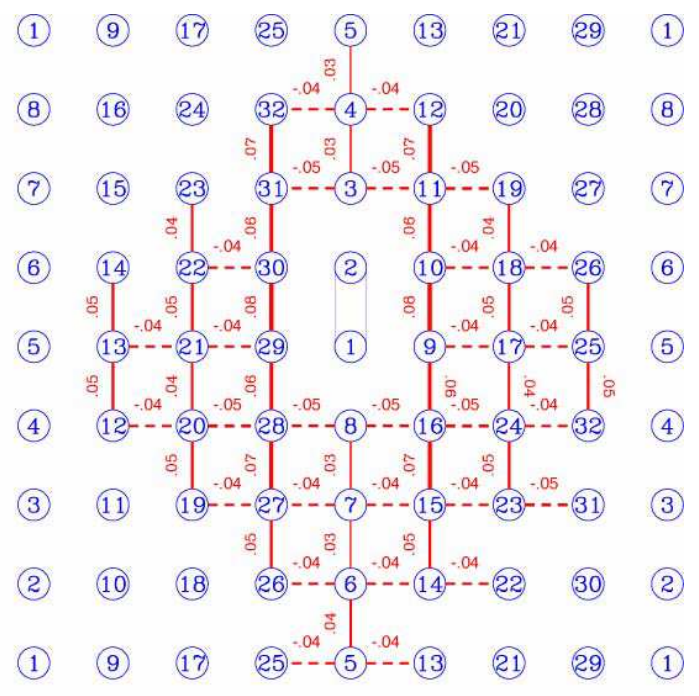

FIG. 5: Bond-nematic correlations of an extended Heisenberg model with $J_{1}=-1, J_{2}=0.4, K=0$, for a cluster of $N=32$ spins, showing the $\mathrm{d}$-wave character of the nematic phase. Correlations are measured relative to the reference bond 1-2.

Although it is hard to put a precise boundary on the stability of the $(\pi, 0)$ collinear AF state, our ED studies suggest that Néel order begins to break down in the FM $J_{1}-J_{2}$ model for $J_{2} \sim 0.6-0.7\left|J_{1}\right|$. We also have performed ED calculations for parameter sets interpolating between the FM $J_{1-} J_{2}$ model for $K=0$ and the FM multiple spin exchange model for $J_{2}=0$. These confirm that the nature of the state bordering the FM phase does not change. Thus a single nematic phase interpolates between the two different Néel order parameters in these two limits, and the FM phase for large $\left|J_{1}\right|$, as shown in Fig. [1]

To conclude - in the frustrated square lattice FM's considered in this letter, the saturated FM ground state undergoes a first order transition into a gapless spinliquid state with bond-nematic order. In applied magnetic field, the transition becomes second order and can be related directly to the condensation of two-magnon bound states.

It is our pleasure to acknowledge stimulating discussions with Andreas Läuchli, Claire Lhuillier and Karlo Penc. Computations were performed at IDRIS (Orsay). This work was supported by Grant-in-Aid for Scientific Research from MEXT of Japan and SFB 463 of the DFG.

[1] P. W. Anderson, Mater. Res. Bull. 8, 153 (1973).

[2] G. Misguich and C. Lhuillier, in "Frustrated spin systems", edited by H.T. Diep, World-Scientific (2004).

[3] K. Ishida et al., Phys. Rev. Lett. 79, 3451 (1997).

[4] R. Masutomi et al., Phys. Rev. Lett. 92, 025301 (2004). 
[5] E. Kaul et al., J. Magn. Magn. Matt. 272-276(II), 922 (2004)

[6] H. Kageyama et al., J. Phys. Soc. Jpn. 74, 1702 (2005)

[7] A. V. Chubukov, Phys. Rev. B 44 (1991), R4693.

[8] T. Momoi, P. Sindzingre and N. Shannon, in preparation.

[9] K. Harada and N. Kawashima, Phys. Rev. B 65, 052403 (2002).

[10] A. Läuchli et al., Phys. Rev. Lett. 95, 137206 (2005).

[11] P. Chandra, P. Coleman and A. I. Larkin, Phys. Rev. Lett. 64, 88 (1990).

[12] M. Roger and J. H. Hetherington, Phys. Rev. B 41, 200 (1990).
[13] N. Shannon et al., Eur. Phys. J. B 38, 599 (2004).

[14] P. Sindzingre, N. Shannon and T. Momoi, in preparation.

[15] D. V. Dmitriev, V. Ya. Krivnov, and A. A. Ovchinnikov, Phys. Rev. B 55, 3620 (1997).

[16] T. Momoi and N. Shannon, Prog. Theor. Phys. Suppl. 159, 72 (2005).

[17] P. Sindzingre, Phys. Rev. B 69, 094418 (2004), and reference therein.

[18] P. Hasenfrantz and F. Niedermayer, Z. Phys. 92, 91 (1993). 